\title{
Erratum and Addendum to "Cartesian Currents, Weak Diffeomorphisms and Existence Theorems in Nonlinear Elasticity", this Archive, Volume 106 (1989): 97-159
}

\section{Mariano Giaquinta, Giuseppe Modica \& Jiří SouČEK}

Our proof of Theorem 1 of [3] p. 107 is not correct since the claim (iii) of Proposition 1 is false. Moreover it is not clear whether the space Cart $\left(\Omega, \mathbb{R}^{N}\right)$ in Definition 1 is closed. Because of this, to minimize functionals in Cart $\left(\Omega, \mathbb{R}^{N}\right)$ becomes unreasonable.

Our idea was to consider the smallest closed set with respect to weak convergence in $\mathscr{C}$ containing the graphs of regular mappings. According to that, the definition of Cart $\left(\Omega, \mathbb{R}^{N}\right)$ has to be changed into the following

Definition 1. Cart $\left(\Omega, \mathbb{R}^{N}\right)$ is the smallest sequentially closed set in $\mathscr{C}$ (with respect to weak convergence of sequences in $\mathscr{C}$ ) containing the graphs of $C^{1}$ mappings from $\bar{\Omega}$ into $\mathbb{R}^{N}$.

Recalling that $T_{k} \overrightarrow{\mathscr{C}}_{\mathscr{G}} T$ if and only if $\left\{T_{k}\right\}$ has equibounded Cart-norm and $T_{k} \rightarrow T$ in the sense of currents, we can say: Cart $\left(\Omega, \mathbb{R}^{N}\right)$ is the sequential closure of $C^{1}$ graphs with respect to the convergence of currents with equibounded Cartnorms; in brief, we shall say that $\operatorname{Cart}\left(\Omega, \mathbb{R}^{N}\right)$ is the weak sequential closure in $\mathscr{C}$ of $C^{1}$ graphs.

Let $M$ be any subset of $\mathscr{C}$ and let $\omega_{1}$ be the first uncountable ordinal. Classically, compare e.g. [1], [4], one can define by transfinite induction the weak sequential closure of $M$ as

where

$$
\mathrm{sw}-\operatorname{cl}(M):=\bigcup_{\alpha<\omega_{1}} M^{(\alpha}
$$

$$
M^{(0)}=M
$$

and $M^{(\alpha)}$, for $\alpha \neq 0$, is defined as follows: if $\alpha$ is a limit ordinal, then

$$
M^{(\alpha)}:=\bigcup_{\beta<\alpha} M^{(\beta)}
$$


if $\alpha=\beta+1$, then

$$
M^{(\alpha)}:=\left\{T \in \mathscr{C} \mid \exists\left\{T_{k}\right\} \subset M^{(\beta)} \text { with } T_{k} \overrightarrow{\mathscr{C}} T\right\} .
$$

By definition we have

$$
\operatorname{Cart}\left(\Omega, \mathbb{R}^{N}\right)=\mathrm{sw}-\mathrm{cl} \text { (graphs of } C^{1} \text { mappings). }
$$

The proofs of the properties of cartesian currents in [3] can be now reread as the first step of the transfinite inductive proof of the same properties for the elements of our newly defined space Cart $\left(\Omega, \mathbb{R}^{N}\right)$. Since the second step of the transfinite induction goes on essentially in the same way, the old proof provides in fact a complete proof of Theorems 2 and 3, where $\operatorname{Cart}\left(\Omega, \mathbb{R}^{N}\right)$ has to be understood in the sense of Definition 1 above. Of course, a priori we do not know whether a current $T$ in $\operatorname{Cart}\left(\Omega, \mathbb{R}^{N}\right)$ can be approximated weakly in $\mathscr{C}$ by a sequence of smooth graphs.

The previous remarks apply to the other spaces defined in [3]: $\operatorname{Dif}(\Omega, \hat{\Omega})$, $\operatorname{Dif}(\Omega), \operatorname{Cart}^{p}\left(\Omega, \mathbb{R}^{N}\right), \operatorname{Dif}^{p, q}(\Omega, \hat{\Omega}), \operatorname{Dif}^{p}(\Omega)$, $\operatorname{Dif}^{p, q}(\Omega)$. Their definitions should be modified accordingly by considering the sequential weak closure in the sense of currents with equibounded relative norms of the respective classes of graphs of smooth mappings or diffeomorphisms.

Actually, following our Remark 1, p. 110 of [3] all properties of the elements of Cart $\left(\Omega, \mathbb{R}^{N}\right)$, and moreover, of all other spaces, can be recovered by showing that the same properties are enjoyed by the elements of the analogous spaces with small initial letters. Since this is not always evident from [3], we shall now supply a few precise statements and proofs we think are needed.

We recall that

$\operatorname{cart}\left(\Omega, \mathbb{R}^{N}\right):=\left\{T \in \mathscr{R}_{n}(U) \mid\|T\|_{\mathscr{G}}<+\infty, p_{\#} T=\llbracket \Omega \rrbracket, \quad T_{\overline{0} 0} \geqq 0, \quad \partial T=0\right\}$.

The following theorem contains essentially Theorems 1 and 2, Section 3 of [3].

Theorem 1. Let $T=\tau(\mathscr{M}, \theta, \xi) \in \operatorname{cart}\left(\Omega, \mathbb{R}^{N}\right)$.

(i) For a.e. $x \in \Omega, p^{-1}(x) \cap \mathscr{M}_{+}$contains exactly one element, denoted by $(x, \tilde{u}(x))$, and $\theta=1 \mathscr{H}^{n}$ a.e. in $\mathscr{M}_{+}$.

(ii) $M_{\beta \bar{\alpha}}(T):=\sigma_{\alpha} p_{\#} T_{\alpha \beta}$ are measures in $\Omega ; u^{j}(T):=p_{\#}\left(T_{\overline{0} 0}\left\llcorner y^{j}\right)\right.$ is absolutely continuous with respect to Lebesgue measure, $u^{j}(T)=: u_{T}^{j}(x) d x$; moreover, $u_{T} \in \operatorname{BV}(\Omega)$ and

$$
\left\|u_{T}\right\|_{L^{1}(\Omega)}=\|T\|_{L^{1}} .
$$

(iii) For all bounded functions $\phi(x, y) \in C^{0}(U)$

$$
T_{\overline{0} 0}\left(y^{j} \phi(x, y)\right)=\int_{\Omega} \phi\left(x, u_{T}(x)\right) u_{T}^{j}(x) d x
$$

and $\tilde{u}=u_{T}$ a.e. in $\Omega$.

(iv) The map $T \rightarrow u_{T}$ defines a continuous immersion of $\operatorname{cart}\left(\Omega, \mathbb{R}^{N}\right)$ into $\mathrm{BV}\left(\Omega, \mathbb{R}^{N}\right)$ in the sense that

$$
\left\|u_{T}\right\|_{\mathrm{BV}\left(\Omega, \mathrm{R}^{N}\right)} \leqq\|T\|_{\mathrm{Cart}\left(\Omega, \mathrm{R}^{N}\right)} \quad \forall T \in \operatorname{cart}\left(\Omega, \mathbb{R}^{N}\right) .
$$


If $T_{k} \underset{\mathscr{G}}{ } T$, then $u_{T_{k}} \rightarrow u$ in $L^{1}\left(\Omega, \mathbb{R}^{N}\right)$ and $D u_{T_{k}} \rightarrow D u_{T}$ in the sense of measures.

(v) The class cart $\left(\Omega, \mathbb{R}^{N}\right)$ is weakly sequentially closed in $\mathscr{C}$; thus

$$
\operatorname{Cart}\left(\Omega, \mathbb{R}^{N}\right) \subset \operatorname{cart}\left(\Omega, \mathbb{R}^{N}\right) .
$$

Proof. We need only to prove (iii) and (v) since the rest has already been proved in Theorems 2 and 3, Section 3 of [3]. From the area formula we have

$$
T_{\overline{0} 0}\left(y^{j} \phi(x, y)\right)=\int_{\Omega} \phi\left(x, \tilde{u}_{T}(x)\right) \tilde{u}_{T}^{j}(x) d x
$$

for all $\phi \in C_{c}^{0}(U)$. Since $\|T\|_{L^{1}}<+\infty$, we get $\|\tilde{u}\|_{L^{1}(\Omega)}<+\infty$; hence (1) holds for all continuous and bounded $\phi$. In particular we deduce

$$
u^{j}(T)(\phi(x))=: \int_{\Omega} \phi(x) u_{T}^{j}(x) d x=\int_{\Omega} \phi(x) \tilde{u}^{j}(x) d x
$$

for all $\phi \in C_{c}^{0}(\Omega)$, i.e., $\tilde{u}=u_{T}$. This proves (iii). In order to prove (v) we observe that

thus

$$
\|T\|_{L^{s}}=\left(\int_{\mathscr{M}}|q(z)|^{s} \xi_{\overline{0} 0}(z) \theta(z) d \mathscr{H}^{n}(z)\right)^{1 / s} \quad s \geqq 1 ;
$$

$$
\left|T_{\overline{0} 0}(\phi(x))\right| \leqq \frac{1}{R}\|T\|_{L^{1}}
$$

for $\quad \phi \in \mathscr{F}_{R}:=\left\{\phi \in C^{0}(U)|| \phi \mid \leqq 1\right.$, spt $\left.\phi \subset \Omega \times\left(\mathbb{R}^{n} \backslash B_{R}\right)\right\}$. Let $\quad \phi \in C^{0}(U)$, $|\phi| \leqq 1, p(\operatorname{spt} \phi) \subset C \Omega, R>0$. We split $\phi$ into $\phi=\phi_{1}+\phi_{2}$ with $\phi_{1} \in C_{c}^{0}(U)$, $|\phi| \leqq 1$ and $\phi_{2} \in \mathscr{F}_{R}$. As

$$
\sup _{k}\left|T_{k \overline{0} 0}\left(\phi_{2}\right)\right| \leqq \frac{1}{R} \sup _{k}\left\|T_{k}\right\|_{L^{1}},
$$

we conclude that $T_{k \overline{0} 0}(\phi) \rightarrow T_{\overline{0} 0}(\phi)$. This proves (iv), since obviously $T_{\overline{0} 0} \geqq 0$, $\partial T=0$, and $T \in \mathscr{R}_{n}(U)$ by the Federer-Fleming closure theorem.

As a consequence of Theorem 1, one easily sees that (except for (i) $)_{1}$ and with (ii) replaced by $\operatorname{spt} T \subset \bar{\Omega} \times \overline{\hat{\Omega}})$ Theorem 4, p. 114 of [3] holds for $T \in \operatorname{dif}(\Omega, \hat{\Omega})$, where

$$
\begin{aligned}
\operatorname{dif}(\Omega, \hat{\Omega}):=\left\{T \in \mathscr{R}_{n}(U) \mid \boldsymbol{M}(T)<+\infty,\right. & p_{\#} T=\llbracket \Omega \rrbracket, q_{\#} T \\
& \left.=\llbracket \hat{\Omega} \rrbracket, \xi_{\overline{0} 0} \geqq 0, \xi_{0 \overline{0}} \geqq 0, \partial T=0\right\} .
\end{aligned}
$$

Also, $\operatorname{dif}(\Omega, \hat{\Omega})$ is closed with respect to the weak convergence of currents with equibounded masses. As the weak sequential closure of $\operatorname{Diff}(\Omega, \hat{Q})$ with respect to $\overrightarrow{\mathscr{C}}_{\mathrm{G}}$ in $\left\{T \in \mathscr{D}_{n}(U) \mid \boldsymbol{M}(T)<+\infty\right\}$, denoted by $\operatorname{Dif}(\Omega, \hat{\Omega})$, is contained in $\operatorname{dif}(\Omega, \hat{\Omega})$, we also see at once that Theorem 4 holds for $T \in \operatorname{Dif}(\Omega, \hat{\Omega})$, too. 
The set of cartesian currents in $\Omega \times \mathbb{R}^{n}$ satisfying

$$
\begin{gathered}
\xi_{0 \overline{0}} \geqq 0, \\
T_{0 \overline{0}}(\phi(x, y)) \leqq \int_{\mathbb{R}_{y}^{n}} \max _{x \in \Omega} \phi(x, y) d y \quad \forall \phi \in C_{c}^{0}(U), \phi \geqq 0
\end{gathered}
$$

is denoted by dif $(\Omega)$. We mention that (2) and (3) essentially appear also in [2].

Proposition 1. Let $T=\tau(\mathscr{M}, \theta, \xi) \in \operatorname{dif}(\Omega)$ and

$$
\mathscr{M}^{+}:=\left\{z \in \mathscr{M} \mid \xi_{00}(z) \geqq 0\right\}
$$

(i) For a.e. $y \in q\left(\mathscr{M}^{+}\right)$there exists exactly one $z \in q^{-1}(y) \cap \mathscr{M}^{+}$, denoted by $\left(\hat{u}_{T}(y), y\right)$; moreover $\mathscr{H}^{n}\left(q(\mathscr{M}) \backslash q\left(\mathscr{M}^{+}\right)\right)=0$, and $\theta=1$ on $\mathscr{M}^{+}$and

$$
\mathscr{M}^{+}=\left\{(\hat{u}(y), y) \mid y \in q\left(\mathscr{M}^{+}\right)\right\}
$$

hold $\mathscr{H}^{n}$ a.e.

(ii) $\operatorname{dif}(\Omega)$ is closed with respect to the weak $\mathscr{C}$-convergence.

Proof. (ii) is trivial. Let us prove (i). From (3) we easily deduce that

$$
T_{0 \overline{0}}(\phi(y)) \leqq \int_{\mathbf{R}_{y}^{n}} \phi(y) d y \quad \forall \phi \in C_{c}^{0}\left(\mathbb{R}_{y}^{n}\right), \phi \geqq 0,
$$

while from the area formula we get for $\phi \geqq 0$

$$
T_{0 \overline{0}}(\phi(y))=\int_{\mathbb{R}_{y}^{n}} \phi(y) \sum_{z \in \mathcal{q}^{-1}(y) \cap \mathscr{A}^{+}} \theta(z) d y \leqq \int_{\mathbb{R}_{y}^{n}} \phi(y) d y .
$$

Thus $\Sigma_{z \in q^{-1}(y) \cap \mathscr{C}^{+}} \theta(z)$ is either 0 or 1 for a.e. $y \in \mathbb{R}_{y}^{n}$; as $\theta \geqq 1$ on $\mathscr{M}$, (i) follows.

We observe that (3) is obviously satisfied for $u \in \operatorname{Diff}(\Omega)$, as it amounts to

$$
\int_{\Omega} \operatorname{det} D u(x) d x \leqq|u(\Omega)|
$$

Consequently, the weak sequential closure in $\mathscr{C}$ of $\operatorname{Diff}(\Omega)$, denoted $\operatorname{Dif}(\Omega)$, is contained in $\operatorname{dif}(\Omega)$.

Let us now discuss the classes $\operatorname{cart}^{p}\left(\Omega, \mathbb{R}^{N}\right)$ and $\operatorname{Cart}^{p}\left(\Omega, \mathbb{R}^{N}\right), \quad p>1$. We set

$$
\operatorname{cart}^{p}\left(\Omega, \mathbb{R}^{N}\right)=\left\{T \in \operatorname{cart}\left(\Omega, \mathbb{R}^{N}\right)\|\| T \|_{\mathscr{C} p}<+\infty\right\}
$$

One easily sees that the $\mathscr{G}^{p}$-norm in [3], also denoted \|\|$_{\text {Cart }}{ }^{p}\left(\Omega, \mathbb{R}^{N}\right)$, is given by

$$
\|T\|_{\mathrm{Cart}^{p}\left(\Omega, \mathbb{R}^{N}\right)}:=\|T\|_{L^{p}}+\|T\|_{M^{p}}
$$


where for $T=\tau(\mathscr{A}, \theta, \xi)$

$$
\begin{aligned}
\|T\|_{L^{p}} & :=\left(\int_{\mathscr{H}}|q(z)|^{p} \xi_{\overline{0} 0}(z) \theta(z) d \mathscr{H}^{n}(z)\right)^{1 / p}, \\
\|T\|_{M^{p}}: & =\left(\int_{\mathscr{H}} \xi_{\overline{0} 0}^{1-p} \theta(z) d \mathscr{H}^{n}(z)\right)^{1 / p} .
\end{aligned}
$$

The following theorem provides a version of Theorem 1 in [3], p. 120 for $T \in$ $\operatorname{cart}^{p}\left(\Omega, \mathbb{R}^{N}\right)$.

Theorem 2. Let $T=\tau(\mathscr{M}, \theta, \xi)$ and $\left\{T_{k}\right\}$ belong to $\operatorname{cart}^{p}\left(\Omega, \mathbb{R}^{N}\right), p>1$. Then we have:

(i) $\xi_{\overline{0} 0}>0 \mathscr{H}^{n}$-a.e. in $\mathscr{M}, \mathscr{M}=\mathscr{M}_{+}$and $\theta=1 \mathscr{H}^{n}$ a.e. Also the measures $M_{\beta \bar{\alpha}}(T):=\sigma_{\alpha} p_{\#} T_{\alpha \beta}$ are absolutely continuous with respect to Lebesgue measure, and

$$
T_{\alpha \beta}(\phi(x, y))=\sigma_{\alpha} \int_{\Omega} \phi\left(x, u_{T}(x)\right) M_{\beta \alpha}^{-}(T)(x) d x
$$

for all bounded Borel functions $\phi(x, y)$.

(ii) Moreover

$$
M_{\beta \bar{\alpha}}(T)(x)=M_{\beta \bar{\alpha}}\left(D u_{T}(x)\right) \quad \text { for a.e. } x \in \Omega
$$

and

$$
\|T\|_{\operatorname{Cart}^{p_{\left(\Omega, \mathbb{R}^{N}\right)}}}=\left\|u_{T}\right\|_{L^{\left.p_{(} \Omega\right)}}+\left\|M\left(D u_{T}\right)\right\|_{L^{p}(\Omega)} .
$$

(iii) $T_{k} \underset{\mathscr{G}^{p}}{\rightarrow} T$ if and only if $u_{T_{k}} \rightarrow u_{T}$ in $L^{p}$ and $M_{\beta \bar{\alpha}}\left(D u_{T_{k}}\right) \rightarrow M_{\beta \bar{x}}\left(D u_{T}\right)$ weakly in $L^{p}$.

(iv) $\operatorname{cart}^{p}\left(\Omega, \mathbb{R}^{N}\right)$ is closed with respect to the weak convergence in $\mathscr{C}^{p}$.

(v) If $\sup _{k}\left\|T_{k}\right\|_{\operatorname{Cart} p_{\left(\Omega, \mathbb{R}^{N}\right)}}<+\infty$ and $u_{T_{k}} \rightarrow w$ weakly* in $L^{1}$, then there exists $T \in \operatorname{cart}^{p}\left(\Omega, \mathbb{R}^{N}\right)$ with $u_{T}=w$ and $T_{k} \underset{\mathscr{G}^{p}}{\rightarrow} T$.

Proof. From

$$
\int_{\mathscr{H}} \xi_{\overline{0} 0}^{1-p} d \mathscr{H}^{n}<+\infty
$$

we get $\xi_{\overline{0} 0}>0$ a.e. in $\mathscr{M}$; thus $\mathscr{M}_{+}=\mathscr{M}$ and $\theta=1 \mathscr{H}^{n}$-a.e. From the area formula, as in Theorem 1 , we deduce that for all $\phi \in C_{c}^{9}(U)$

$$
T_{\alpha \beta}(\phi(x, y))=\int_{p_{\text {井 }}\left(\mathscr{A}_{+}\right)} \phi\left(x, u_{T}(x)\right) \frac{\xi_{\alpha \beta}\left(x, u_{T}(x)\right)}{\xi_{\overline{0} 0}\left(x, u_{T}(x)\right)} d x .
$$

As

$$
\frac{\xi_{\alpha \beta}\left(x, u_{T}(x)\right)}{\xi_{\overline{0} 0}\left(x, u_{T}(x)\right)} \in L^{1}(\Omega)
$$


we deduce that (7) holds for all bounded Borel functions $\phi(x, y)$, and therefore

$$
\sigma_{\alpha} M_{\beta \alpha}(T)(x)=\frac{\xi_{\alpha \beta}\left(x, u_{T}(x)\right)}{\xi_{\overline{0} 0}\left(x, u_{T}(x)\right)} \quad \mathscr{H}^{n} \text {-a.e. in } \Omega .
$$

On the other hand, Theorem 1 yields

$$
M_{i i}(T)(x)=D_{i} u_{T}^{j}(x),
$$

thus (5) follows since $T$ is rectifiable (compare [3], p. 122). Finally,

$$
\int_{\mathscr{H}} \xi_{\overline{0} 0}^{1-p} d \mathscr{H}^{n}=\int_{\Omega} \xi_{\overline{0} 0}\left(x, u_{T}(x)\right)^{-p} d x=\int_{\Omega}\left|M\left(D u_{T}\right)\right|^{p} d x,
$$

and so we have proved (i) and (ii). The claims (iii), (iv), (v) follow from (4) and Theorem 1 (compare also [3], pp. 122-123).

If we now redefine $\operatorname{Cart}^{p}\left(\Omega, \mathbb{R}^{N}\right), p>1$, as the weak sequential closure in $\mathscr{C}^{p}$ of $C^{1}$ graphs, we see at once that Theorem 1, p. 120 of [3] is correct. In fact $\operatorname{Cart}^{p}\left(\Omega, \mathbb{R}^{N}\right)$ is then a sequentially closed subset of $\operatorname{cart}^{p}\left(\Omega, \mathbb{R}^{N}\right)$, thus Theorem 2 above holds in $\operatorname{Cart}^{p}\left(\Omega, \mathbb{R}^{N}\right)$, too. Again, the approximation property by smooth graphs cannot be in general deduced; hence Remark 1, p. 123 of [3] is not correct.

Finally let us reconsider the class of weak diffeomorphisms. It is easily seen that Theorem 2 of [3], p. 125 holds for $T$ in

$$
\operatorname{dif}^{p, q}(\Omega, \hat{\Omega}):=\left\{T \in \operatorname{dif}(\Omega, \hat{\Omega}) \mid\|T\|_{\mathscr{D}, q}<+\infty\right\}
$$

provided we replace the spaces in the statement with the same spaces with small initial letters. Obviously $\operatorname{dif}^{p, q}(\Omega, \hat{\Omega})$ is closed with respect to weak convergence in $\mathscr{D}^{p, q}$. Consequently, Theorem 2 also holds in $\operatorname{Dif}^{p, q}(\Omega, \hat{\Omega})$ defined as the weak sequential closure of $\operatorname{Diff}(\Omega, \hat{\Omega})$ in $\mathscr{D}^{p, q}$. We finally observe that for $T=\tau(\mathscr{M}, \theta, \xi)$

$$
\|T\|_{\mathscr{O} P, q}=\|T\|_{\mathrm{Cart}^{p}\left(\Omega, \mathrm{R}^{n}\right)}+\left(\int_{\mathscr{M}} \xi_{0 \overline{0}}^{1-q} \theta d \mathscr{H}^{n}\right)^{1 / q}
$$

Now, for $p, q>1$, set

$$
\begin{aligned}
\operatorname{dif}^{p}(\Omega) & :=\left\{T \in \operatorname{dif}(\Omega) \mid\|T\|_{\operatorname{Cart}^{p}\left(\Omega, \mathbb{R}^{n}\right)}<+\infty\right\}, \\
\operatorname{dif}^{p, q}(\Omega) & :=\left\{T \in \operatorname{dif}(\Omega) \mid\|T\|_{\mathscr{D}, q}<+\infty\right\} .
\end{aligned}
$$

By definition, these spaces are sequentially weakly closed respectively for $\overrightarrow{\mathscr{C} P}$ and $\underset{\mathscr{P} p, q}{ }$. According to the above, we redefine $\operatorname{Dif}^{p}(\Omega)$ and $\operatorname{Dif}^{p, q}(\Omega)$ as the weak sequential closure of $\operatorname{Diff}(\Omega)$ respectively for weak convergence in $\operatorname{dif}(\Omega) \cap$ $\operatorname{Cart}^{p}\left(\Omega, \mathbb{R}^{n}\right)$ and in $\mathscr{D}^{p, q}$. Then we clearly have

$$
\begin{gathered}
\operatorname{Dif}^{p}(\Omega) \subset \operatorname{dif}^{p}(\Omega), \\
\operatorname{Dif}^{p, q}(\Omega) \subset \operatorname{dif}^{p, q}(\Omega) .
\end{gathered}
$$


The two following theorems are a slightly stronger version of Theorems 3 and 4 of [3] Section 4, and, because of (8), they hold respectively in $\operatorname{Dif}^{p}(\Omega)$ and $\operatorname{Dif}^{p, q}(\Omega)$. too.

Theorem 3. Let $T=\tau(\mathscr{M}, \theta, \xi)$ and $\left\{T_{k}\right\}$ belong to $\operatorname{dif}^{p}(\Omega)$.

(i) Set $u_{T}(\Omega):=q(\mathscr{M})$. Then we have

$$
q_{\#} T=\llbracket u_{T}(\Omega) \rrbracket
$$

and for all bounded Borel functions $\phi(y)$ in $\mathbb{R}_{y}^{n}$

$$
\int_{u_{T}(\Omega)} \phi(y) d y=\int_{\Omega} \phi\left(u_{T}(x)\right) \operatorname{det} D u_{T}(x) d x .
$$

(ii) $\left|u_{T}(\Omega)\right| \leqq|\Omega|^{1 / p^{\prime}}\|T\|_{\mathrm{Cart}^{p_{(}\left(\Omega, \mathrm{R}^{n}\right)}}$.

(iii) If $T_{k} \rightarrow T$, then

$$
\mathscr{H}^{n}\left(u_{T_{k}}(\Omega) \triangle u_{T}(\Omega)\right)=0
$$

where $A \triangle B$ denotes the symmetric difference of $A$ and $B$.

Proof. Using the area formula and Proposition 1, we have

$$
\begin{aligned}
\int_{u_{T}(\Omega)} \phi(y) d y & =\int_{\mathscr{M}^{+}} \phi(y) \xi_{0 \overline{0}}(x, y) d \mathscr{H}^{n}(x, y) \\
& =\int_{\mathscr{H}^{\prime}} \phi(y) \frac{\xi_{0 \overline{0}}(x, y)}{\xi_{\overline{0} 0}(x, y)} \xi_{\overline{0} 0}(x, y) d \dot{\mathscr{H}}^{n}(x, y)=\int_{\Omega} \phi\left(u_{T}(x)\right) \operatorname{det} D u_{T}(x) d x .
\end{aligned}
$$

Hence (i) follows.

(ii) Using the Hölder inequality, we get

$$
\left|u_{T}(\Omega)\right|=\int_{\mathscr{H}} \xi_{0 \overline{0}} d \mathscr{H}^{n} \leqq\left(\int_{\mathscr{H}} \xi_{\overline{0} 0} d \mathscr{H}^{n}\right)^{\frac{1}{p^{\prime}}}\left(\int_{\mathscr{H}} \frac{\xi_{0 \overline{0}}^{p}}{\xi_{\overline{0} 0}^{p-1}} d \mathscr{H}^{n}\right)^{\frac{1}{p}} \leqq|\Omega|^{\frac{1}{p^{\prime}}}\|T\|_{\operatorname{Cart}^{p}\left(\Omega, \mathbb{R}^{n}\right)^{n}} .
$$

(iii) From Theorem 2 we have $q_{ \pm \neq} T_{k}(\phi) \rightarrow q_{\#} T(\phi)$ for all continuous and bounded $\phi$; as $q_{\#} T=\llbracket u_{T}(\Omega) \rrbracket$ we can conclude

$$
\int \phi(y) \chi_{u_{T_{k}}(\Omega)} d y \rightarrow \int \phi(y) \chi_{u_{T}(\Omega)} d y \quad \forall \phi \in C^{0}\left(\mathbb{R}_{y}^{n}\right), \phi \text {. bounded. }
$$

Using this for $\phi=1$, we get $\left|u_{T_{k}}(\Omega)\right| \rightarrow\left|u_{T}(\Omega)\right|$. Because $\left\|\chi_{u_{T^{(\Omega)}}}\right\|_{L^{2}}=\left|u_{T}(\Omega)\right|$ and that $\chi_{u_{T_{k}}(\Omega)} \rightarrow \chi_{u_{T}(\Omega)}$ in $L^{2}$, (iii) follows at once.

Theorem 4. Let $T=\tau(\mathscr{M}, \theta, \xi)$ and $T_{k}$ belong to $\operatorname{dif}^{p, q}(\Omega), p, q>1$.

(i) We have $\xi_{0 \overline{0}}>0$ a.e. in $\mathscr{M}$. Also

$$
\mathscr{H}^{n}\left(u_{T}(\Omega)\right) \geqq \mathscr{H}^{n}(\Omega)^{q^{q}}\|T\|_{\mathscr{O} P, q}^{-1} .
$$

(ii) Let $\hat{u}_{T}$ be the function defined in Propositon 1. For a.e. $y \in u_{T}(\Omega)$ we define the derivatives $D \hat{u}_{T}$ by

$$
D_{j} \hat{u}_{T}^{i}(y):=\frac{\xi_{i j}\left(\hat{u}_{T}(y), y\right)}{\xi_{00}\left(\hat{u}_{T}(y), y\right)} .
$$


Then we have $\hat{u}_{T}, D \hat{u}_{T} \in L^{q}\left(\hat{\Omega_{T}}\right)$.

(iii) Finally

$$
\begin{gathered}
D \hat{u}_{T}\left(u_{T}(x)\right) D u_{T}(x)=\left.\mathbb{1}\right|_{\mathbb{R}^{n}}, \quad D u_{T}\left(\hat{u}_{T}(y)\right) D \hat{u}_{T}(y)=\left.\mathbb{1}\right|_{\mathbb{R}^{n}} \\
M_{\alpha \bar{\beta}}\left(D \hat{u}_{T}(y)\right)=\sigma_{\alpha} \sigma_{\beta} \frac{M_{\beta \bar{\alpha}}\left(D u_{T}\left(\hat{u}_{T}(y)\right)\right)}{\operatorname{det} D u_{T}\left(\hat{u}_{T}(y)\right)}
\end{gathered}
$$

for a.e. $y \in u_{T}(\Omega)$, and

$$
T_{\alpha \beta \beta}(\phi)=\sigma_{\beta} \int_{u_{T}(\Omega)} \phi\left(\hat{u}_{T}(y), y\right) M_{\alpha \bar{\beta}}\left(D \hat{u}_{T}(y)\right) d y
$$

holds for all bounded Borel functions in $U$.

Proof. From

$$
\int_{\mathscr{H}} \xi_{0 \overline{0}}^{1-q} \theta d \mathscr{H}^{n}<+\infty
$$

we deduce that $\xi_{0 \overline{0}}>0$ a.e. in $\mathscr{M}$. As $\theta=1$ a.e. in $\mathscr{M}$

$$
\begin{aligned}
|\Omega| & =\int_{\mathscr{H}} \xi_{\overline{0} 0} d \mathscr{H}^{n} \leqq\left(\int_{\mathscr{H}} \xi_{0 \overline{0}} d \mathscr{H}^{n}\right)^{1 / q^{\prime}}\left(\int_{\mathscr{H}} \frac{\xi_{\overline{0} 0}^{q}}{\xi_{0 \overline{0}}^{q-1}} d \mathscr{H}^{n}\right)^{1 / q} \\
& \leqq\left|u_{T}(\Omega)\right|^{1 / q^{\prime}}\|T\|_{\mathscr{D} p, q} .
\end{aligned}
$$

The proof can now be completed as in Theorem 2 .

With the new definitions of the spaces given in this erratum, all statements and proofs of [3], Sections 5,6,7 hold true with the same proofs.

\section{References}

1. S. Banach, Théorie des opérations linéaires, Warsawa, 1932.

2. P. J. CIARLET \& J. NeČAS, Unilateral problems in nonlinear, three-dimensional elasticity, Arch. Rational Mech. Anal. 97 (1987) 171-188.

3. M. Giaquinta, G. ModicA, \& J. SoučEk, Cartesian currents, weak diffeomorphisms and existence theorems in nonlinear elasticity, Archive for Rational Mech. Anal. 106 (1989) 97-159.

4. I. P. NAtanson, Theory of functions of a real variable, Vol. 2, New York, 1964.

Istituto di Matematica Applicata

Università di Firenze

and

Československá Akademie

Věd Matematický Ústav

Praha 